\title{
DETECTING CELL ASSEMBLY INTERACTION PATTERNS VIA BAYESIAN BASED CHANGE-POINT DETECTION AND GRAPH INFERENCE MODEL
}

\author{
Zhichao Lian ${ }^{1 *}$, Xiang Li $^{2^{*}}$, Hongmiao Zhang ${ }^{3 *}$, Hui Kuang ${ }^{3}$, Kun Xie $^{3}$, Jianchuan Xing ${ }^{4,1}$, Dajiang Zhu ${ }^{2}$, Joe Z. Tsien ${ }^{3 * *}$, \\ Tianming Liu ${ }^{2 * *}$, Jing Zhang ${ }^{1 * *}$
}

\begin{abstract}
${ }^{1}$ Department of Statistics, Yale University, New Haven, CT; ${ }^{2}$ Cortical Architecture Imaging and Discovery Lab, Department of Computer Science, University of Georgia, Athens, GA; ${ }^{3}$ Brain and Behavior Discovery Institute, Georgia Regents University, Augusta, GA; ${ }^{4}$ School of Computer Science and Engineering, University of Electronic Science and Technology of China, Chengdu, P.R. China

*Joint first authors, **Joint corresponding authors.
\end{abstract}

\begin{abstract}
Recent studies have proposed the theory of functional network-level neural cell assemblies and their hierarchical organization architecture. In this study, we first proposed a novel Bayesian binary connectivity change point model to be applied on the binary spiking time series recorded from multiple neurons in the mouse hippocampus during three different emotional events, to find stable temporal segments of neural activity. We then applied a Bayesian graph inference algorithm on the segmentation results to find multiple functional interaction patterns underlying each experience. The resulting interaction patterns were analyzed by multi-view co-training method to identify the common sub-network structure of cell assemblies which are strongly connected i.e. "neural cliques". By analyzing the resulting sub-networks from three memory-producing events, it is found that there exist certain common neurons participating in the functional interactions across different events, lending strong support evidence to the hypothesis of hierarchical organization architecture of neuronal assemblies.
\end{abstract}

Index Terms - neuronal code, cell assebmly interaction

\section{INTRODUCTION}

Recently there have been increasing number of studies on the neuronal network-level organization mechanisms of the brain [1-3], based on the simultaneous monitoring of neural spiking activities across multiple neurons $[4,5]$. These researches have led to a series of exciting results including that ensembles of neurons activation i.e. "population code" are directly correlated with the brain space recognition [6] and memory formation [3, 7]. Also, it has been found that the functions of neural cell assemblies have a hierarchical architecture that enables the brain in dealing with both general and specific memory coding [2]. However, most of the researches utilize the neuron firing rate for characterizing the neural network pattern and the corresponding cell assemblies, and there have been very few studies on inferring the functional interaction patterns and their dynamics between neurons by their spiking time series.

Recently, Bayesian partition model has been widely applied on biomedical data [8-9], including neuroimaging studies [10-12]. Inspired by these studies, in this work, we developed a novel Bayesian network change point model to detect the abrupt changes in neuron spiking time series. The change points were determined by the joint probability among neurons between different time segments, and Markov chain Monte Carlo (MCMC) was applied to sample the posterior probability distribution of each time point being a change point. Based on the detected change points and their corresponding temporal blocks, we further analyzed the functional interaction patterns among 195 neurons by a multi-view co-training method and identified the common neural clique structures which are strongly connected. The major findings of this work are: 1) There exists functional interaction dynamics in response to external stimuli events; 2) Strongly functional interacting neurons form one or more "neural cliques" for a specific event. These results offer supporting evidence to the hypothesis of hierarchical organization architecture of neuronal assemblies [1-3].

\subsection{Data acquisition and pre-processing}

Neural spike data were collected using 128-channel recording arrays recording from the anterior cingulate cortex (ACC) of freely behaving mice. The detailed procedures were previously described in $[5,13]$. In brief, two bundles of 64-channel stereotrodes were implanted in ACC bilaterally $(0.5 \mathrm{~mm}$ anterior to bregma, $0.5 \mathrm{~mm}$ lateral and $1.1-1.2 \mathrm{~mm}$ ventral to the brain surface). Spike activities were recorded via Plexon neural data acquisition system while the animal was subjected to mild blast, air puff and shake stimuli $[5,13]$. The recorded spike activities were sorted into putative single units offline by using MClust 3.3 after automatic pre-clustering with KlustaKwik 1.5. (http://redishlab.neuroscience.umn.edu-

/MClust/MClust.html). The spiking time of totally 195 
neurons were recorded during an 8-minutes period in which the animal was given each stimulus. As the recorded spiking time was point-process data, we used $100-\mathrm{ms}$ time bin to sample the spiking and convert it into binary time series data. Among a total of 4800 bins ( 8 minutes), each 100 -ms time bin was set to the value of 1 if there was at least one neural spiking in that bin, otherwise it was set to the value of 0 . In this way we obtained three binary neuronal activation matrix with the dimension of 195 (neurons) by 4800 (time bins), which would be used for further analysis.

\subsection{Bayesian binary connectivity change point model}

As the input data obtained in 2.1 is a binary multivariate random process, for the set of binary column vectors $x_{1}, x_{2}, \ldots, x_{T}$ i.i.d. (independent and identically distributed) from the $m$-dependant binary random variable probability mass function we have:

$$
x_{t} \sim p=\left(p_{1}, p_{2}, \ldots, p_{2^{m}}\right)^{T}, \quad \mathrm{t}=1,2, \ldots, \mathrm{T}
$$

where $T$ is the number of vectors, $m$ is the dimension of vector, $2^{m}$ is the total number of possible combinations for $m$-dependant binary random variables, and $p_{1}, \ldots, p_{2^{m}}$ are the probabilities of all combinations with sum of 1 . The likelihood of $x_{1}, x_{2}, \ldots, x_{T}$ given $p_{1}, \ldots, p_{2^{m}}$ is:

$$
P\left(x_{1}, x_{2}, \ldots, x_{T} \mid p_{1}, \ldots, p_{2^{m}}\right)=\prod_{j=1}^{2^{m}} p_{j}^{n_{j}}
$$

where $n_{j}$ is the number of column vectors in $x_{1}, x_{2}, \ldots, x_{T}$ taking the $j$-th combination $j=1,2, \ldots, 2^{m}$. As $p_{1}, \ldots, p_{2^{m}}$ are unknown, we assume they are random and apply Dirichlet prior on them:

$$
\begin{aligned}
& p \sim \operatorname{Dirichlet}\left(\alpha_{1}, \ldots, \alpha_{2^{m}}\right): P\left(p_{1}, \ldots, p_{2^{m}} \mid \alpha_{1}, \ldots, \alpha_{2^{m}}\right)=\frac{1}{B(\alpha)} \prod_{j=1}^{2^{m}} p_{j}^{\alpha_{j}-1} \\
& B(\alpha)=\frac{\prod_{j=1}^{2^{m}} \Gamma\left(\alpha_{j}\right)}{\Gamma\left(\sum_{j=1}^{2^{m}} \alpha_{j}\right)}, \alpha=\left(\alpha_{1}, \ldots, \alpha_{2^{m}}\right), \Gamma(x)=\int_{0}^{\infty} t^{x-1} e^{-t} d t
\end{aligned}
$$

Thus:

$$
\begin{aligned}
P\left(x_{1}, x_{2}, \ldots, x_{T}, p_{1}, \ldots, p_{2^{m}}\right) & =\prod_{j=1}^{2^{m}} p_{j}^{n_{j}} \times \operatorname{Dirichlet}\left(\alpha_{1}, \ldots, \alpha_{2^{m}}\right) \\
& =\frac{1}{B(\alpha)} \prod_{j=1}^{2^{m}} p_{j}^{n_{j}+\alpha_{j}-1}
\end{aligned}
$$

By integrating $p$, we have the probability of $x_{1}, x_{2}, \ldots, x_{T}$ :

$$
\begin{aligned}
P\left(x_{1}, x_{2}, \ldots, x_{T}\right) & =\int P\left(x_{1}, x_{2}, \ldots, x_{T}, p\right) d p \\
& =\prod_{j=1}^{2^{m}} \frac{\Gamma\left(n_{j}+\alpha_{j}\right)}{\Gamma\left(\alpha_{j}\right)} \frac{\Gamma\left(\sum_{j=1}^{2^{m}} \alpha_{j}\right)}{\Gamma\left(\sum_{j=1}^{2^{m}}\left(n_{j}+\alpha_{j}\right)\right)}
\end{aligned}
$$

where we take $\alpha_{1}=\alpha_{2}=\ldots=\alpha_{2^{m}}=1 / 2^{m}$.

Given an $m \times T$ neuron spiking matrix $\mathbf{X}=\left(x_{1}, x_{2}, \ldots, x_{T}\right)$ where $m$ is the number of neurons and $T$ is the number of observations in temporal order, a block indicator vector
$I=\left(I_{1}, I_{2}, \ldots, I_{T}\right)$ is defined to indicate the possible locations of network change points, where $I_{t}=1$ indicates that there is a change point at time point $t$ and there is a new block, $I_{t}=0$ otherwise. The time serials matrix $\mathbf{X}$ is thus divided into $\Sigma_{t=1}^{T} I_{t}$ blocks. $I_{l}$ is always 1 , as the first time point is always the beginning of the first block. The likelihood of the neuron spiking matrix $\mathbf{X}=\left(x_{1}, x_{2}, \ldots, x_{T}\right)$ is then:

$$
p(\mathbf{X} \mid I)=\prod_{b=1}^{\sum_{t} I_{t}} p\left(\mathbf{X}_{\mathbf{b}}\right)
$$

where $\mathbf{X}_{\boldsymbol{b}}$ are the temporal data belonging to the $b$-th block and $\mathrm{p}\left(\mathbf{X}_{\boldsymbol{b}}\right)$ is calculated according to Eq. (5). We assume that the temporal blocks are independent from each other, therefore the posterior distribution $p(I \mid \mathbf{X})$ satisfies

$$
p(I \mid \mathbf{X}) \propto p(I) p(\mathbf{X} \mid I)
$$

where $p(I)=\prod_{t=1}^{T} p\left(I_{t}\right)$ and $p\left(I_{t}\right) \sim \operatorname{Bern}(0.5)$.

We then design a Markov chain Monte Carlo (MCMC) scheme [14] to sample the posterior distribution $p(I \mid \mathbf{X})$ with a random initial block indicator vector $I^{0}$. For $i$-th iteration:

1. A new block indicator vector $I^{*}$ is proposed by randomly choosing an indicator in $I^{i-1}$ and its value is changed from 0 to 1 or from 1 to 0 .

2. $p\left(I^{*} \mid \mathbf{X}\right)$ is then evaluated by Eq. (7).

3. A uniform $(0,1)$ random number $u$ is generated and set:

$$
I^{i}=\left\{\begin{array}{lr}
I^{*} & \text { if } u \leq \min \left[1, \frac{p\left(I^{*} \mid \mathbf{X}\right)}{p\left(I^{i-1} \mid \mathbf{X}\right)}\right] \\
I^{i-1} & \text { otherwise }
\end{array}\right.
$$

4. Iterate until $i$ reaches a given number $N$ (in this work, $N$ is set as 2000 to make sure that $p\left(I^{i} \mid \mathbf{X}\right)$ converges).

5. Finally, we exclude the burn-in from the MCMC samples and calculate the posterior probability for each time point to be a change point from MCMC samples.

\section{RESULTS}

\subsection{Results on neuronal recording dataset}

In this work, we have applied the proposed model on the three time series neural spiking dataset obtained as described in 2.1. Taken the results from blast event data as an example, the timings of the 8 change points, along with the timings of the blast stimulus are depicted in Fig. 1. According to the change points vs. stimulus timing plot, it could be concluded that the change points detected are well in correspondence with the stimulus. Such correspondence validates the practicability of our model as it has been shown in previous literature that both single neuronal and network-level neuronal activation are expected to respond to external inputs [1,7]. Moreover, previously the response was mainly characterized by the change of the activity patterns of neuron(s), e.g. by the firing rate [7]. However, the change points detected by our model capture the dynamics of the functional interaction patterns among 
neural cell assembly, inferred by a Bayesian basis. Thus the aligned change point/stimuli pairs imply the dynamic nature of the brain functional organization mechanism at neuronal level, i.e. there exists a perpetual "functional switching" in the brain where functional networks across regions are dynamic, subjecting to the external input, its own previous states and other contexts.

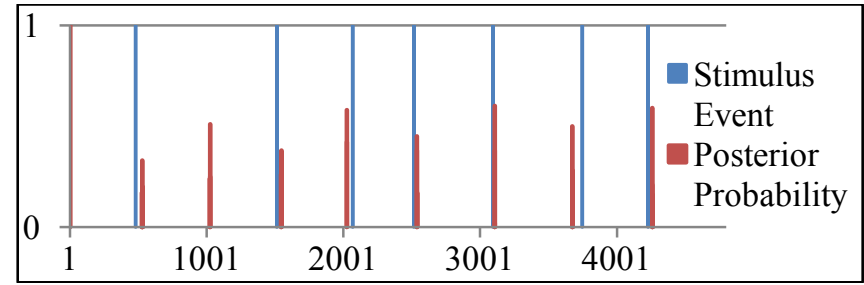

Figure 1. Posterior probability (on y-axis) of each temporal position (on x-axis) to be a change point during blast event, colored in red. Also, the starting point of each repeat of the stimulus is colored in blue. It should be noted that the red lines in the figure are not deterministic, and the change points could be existing within a small range around.

\subsection{Functional interaction patterns analysis}

Based on the detected change points and corresponding temporal blocks defined by them, we have used the widelyapplied GES (Greedy Equivalence Search) Bayesian graph algorithm [15] on the neural spiking time series segmented by the temporal blocks to infer the functional interaction patterns represented by the causal graphs from the data. Again, taking the blast event for example, as there were totally 8 change points detected, we have obtained 9 functional interaction patterns $P_{1}, \ldots, P_{9}$, each of which is a $195 * 195$ connectivity matrix showing the causal links between variable pairs, as illustrated in Fig. 2a. Similar methods were applied on the dataset from other two events (air puff, shake) as well. Further, in this work we are interested in the common sub-structures across interaction patterns of each event, which characterizes the consistent functional interaction structure maintained during the event. To achieve this goal, for each of the three event, we applied the graph based multi-view co-training method [16] on the interaction patterns obtained from it, aiming to maximize the agreement across different patterns. In short, the method takes the similarity matrix from each view and tries to project all of them along the $k$ eigenvectors obtained from their graph Laplacian. Then it inversely projects the results to obtain each modified ("trained") similarity matrix. The procedure iteratively performs the projection/inverseprojection by $n$ steps, finally obtaining several consistent clusters of the similarity matrix with maximizing inter cluster discrimination ability, while discarding the local information of individual matrices. In this work, we consider the connectivity matrices obtained from different temporal segments as different "views" of the interaction pattern of the whole event, and the clusters in the training result as the consistently participating neuronal subnetworks that are stable over time. The training result of the blast event is visualized in Fig. 2b. It could be seen that the 195 neurons formed two clear clusters (sub-networks). There is strong inter-neuron functional interaction within each sub-network which almost constituted two complete graphs i.e. neural clique assemblies. The concept of neural clique has been observed and reported in previous literature based on the co-firing of the neurons [1]. However, in this work the clique is formed by identifying the common substructure of the inferred functional interaction patterns.

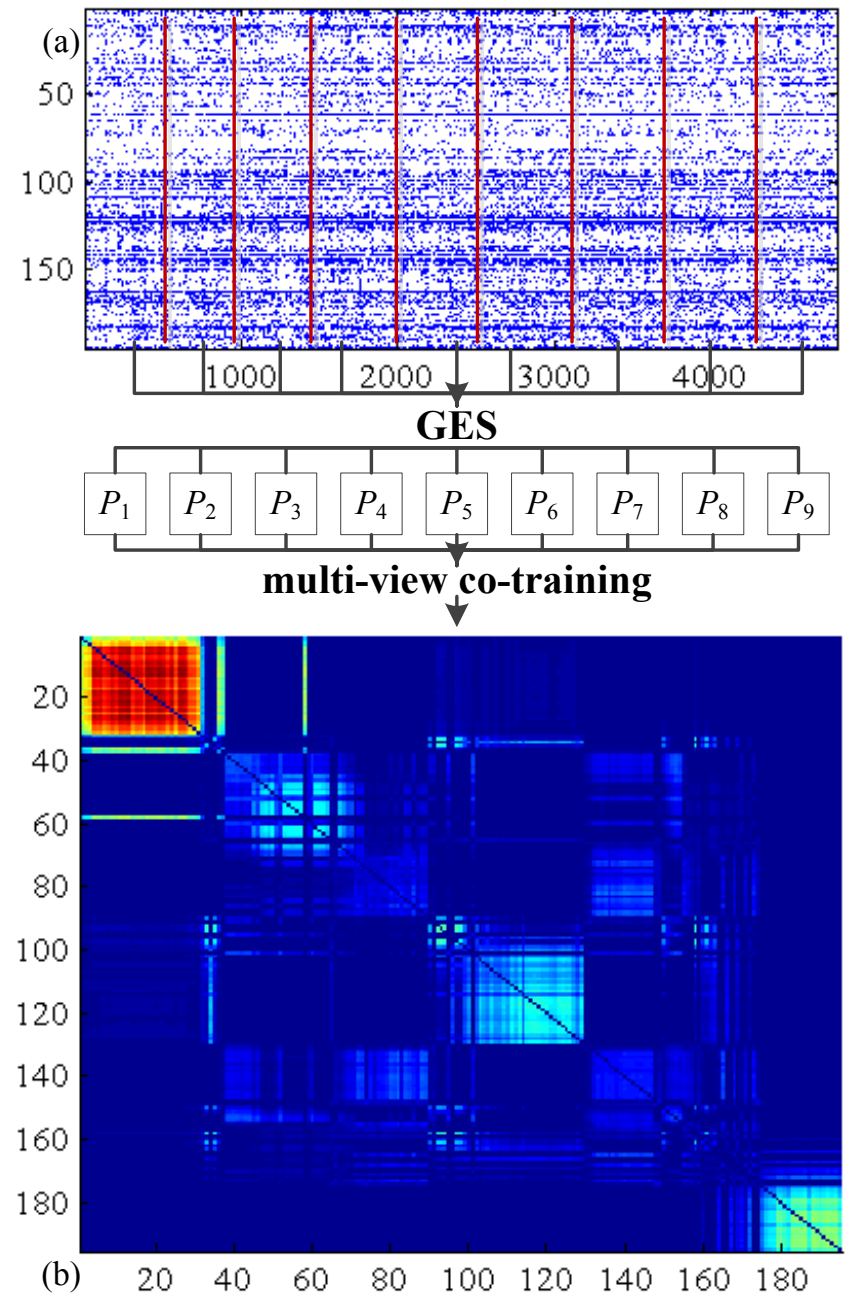

Figure 2. (a) Binary time series of the neural spiking data during blast event, red bars indicate the detected change points. Through GES inference, $9195^{*} 195$ connectivity matrices were obtained, marked as $P_{1}$ to $P_{9}$ in the figure. (b) The multi-view co-training result from the 9 GES results, which is also a $195 * 195$ connectivity matrix, color coded by the intensity of interaction. The indices of neurons were re-ordered so that the bandwidth of the matrix is minimized.

Using the similar procedure, we have obtained the multi-view co-training results characterizing the consistent interaction patterns during the air puff and shake events as well, which are shown in Fig. $3 \mathrm{a}$ and $3 \mathrm{~b}$. It could be observed that similar to the blast event, neurons were organized into smaller but strongly interconnected sub- 
networks shown as the bright-colored blocks in the matrix visualizations. If we focus on the most strongly connected sub-networks shown as the red blocks, it would be found that the sizes of those sub-networks are similar across events. In the blast event shown in Fig. 2b, there were 36 neurons strongly connected and formed the clique (red block), while in the air puff event (Fig. 3a) there were 35 neurons, and in shake event (Fig. 3b) there were 33 neurons. Further, we summarized the common neurons that participated in those most strongly connected sub-networks across different tasks, i.e., the intersection among the three cliques, and found that there were 13 common neurons involved in the subnetworks of the blast and air puff events, 9 neurons in air puff and shake events, and 6 neurons in blast and shake events, shown as in Fig. 3c. Out of those common neurons between two events, there were 3 neurons that participated in the sub-networks of all three events with index \#29, \#35 and \#144. Such findings revealed the possibility that neuronal functional interaction might be hierarchically organized, in correspondence with previous literature reports [2], where certain "general" neurons would be highly interacting with other neurons in different tasks.

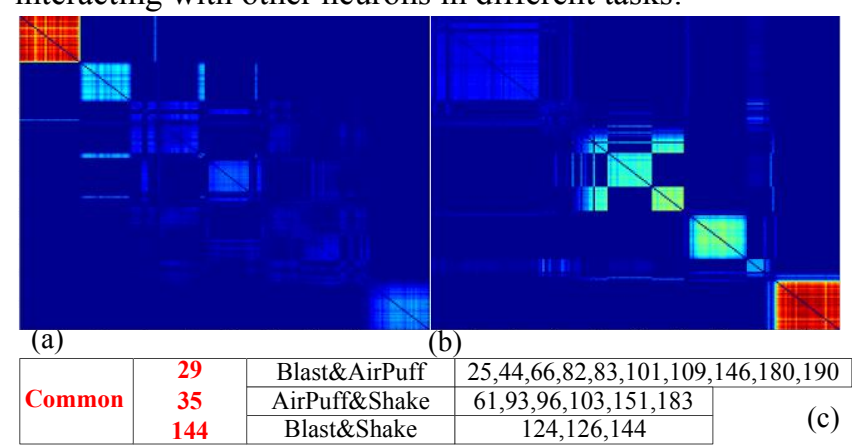

Figure 3. (a) The multi-view co-training result from the GES results of air puff event. (b) The multi-view co-training result from the GES results of shake event. It should be noted that the neurons have been re-ordered thus there are no correspondence between the indices across events. (c) Indices of the common neurons across all three events (marked by "Common") and across two events (marked by event names).

\section{DISCUSSION AND CONCLUSION}

In this work, we have proposed a novel Bayesian binary connectivity change point model to detect the change points from neural spiking data and obtained reasonable results. The strong correspondence between the results from our model and from previous studies has shown that there is a potentially novel way in discovering neural encoding patterns from a dynamic interaction perspective. As no apriori input is needed for our model, we could further apply it on time periods during which no explicit external stimuli is given i.e. resting-state or cognitive state and study their internally-driven functional organization patterns.

\section{REFERENCES}

[1] L. Lin, R. Osan, S. Shoham et al., "Identification of network-level coding units for real-time representation of episodic experiences in the hippocampus," Proceedings of the National Academy of Sciences, vol. 102, no. 17, pp. 6125-6130, April 26, 2005.

[2] L. Lin, R. Osan, and J. Z. Tsien, “Organizing principles of real-time memory encoding: neural clique assemblies and universal neural codes," Trends in Neurosciences, vol. 29, no. 1, pp. 48-57, 1, 2006.

[3] G. Chen, L. P. Wang, and J. Z. Tsien, "Neural Population-Level Memory Traces in the Mouse Hippocampus," PLoS ONE, vol. 4, no. 12, pp. e8256, 2009.

[4] G. Buzsaki, "Large-scale recording of neuronal ensembles," Nat Neurosci, vol. 7, no. 5, pp. 446-451, 2004 .

[5] H. Kuang, and J. Tsien, "Large-Scale Neural Ensembles in Mice: Methods for Recording and Data Analysis," Electrophysiological Recording Techniques, Neuromethods R. P. Vertes and R. W. Stackman Jr, eds., pp. 103-126: Humana Press, 2011.

[6] M. Wilson, and B. McNaughton, "Dynamics of the hippocampal ensemble code for space," Science, vol. 261, no. 5124, pp. 1055-1058, August 20, 1993.

[7] S. Wirth, M. Yanike, L. M. Frank et al., "Single Neurons in the Monkey Hippocampus and Learning of New Associations," Science, vol. 300, no. 5625, pp. 15781581, June 6, 2003.

[8] Z. Lian, Q. N. Tian, Y. Liu et al., "Detecting hepatitis B viral amino acid sequence mutations in occult hepatitis B infections via Bayesian partition model," Journal of Proteomics \& Bioinformatics, 2013.

[9] J. Zhang, T. Hou, W. Wang et al., "Detecting and understanding combinatorial mutation patterns responsible for HIV drug resistance," Proceedings of the National Academy of Sciences, January 11, 2010.

[10] J. Zhang, X. Li, C. Li et al., "Inferring Functional Interaction and Transition Patterns via Dynamic Bayesian Variable Partition Models," Human Brain Mapping, in Press. 2013.

[11] X. Li, D. Zhu, X. Jiang et al., "Dynamic functional connectomics signatures for characterization and differentiation of PTSD patients," Human Brain Mapping, 2013.

[12] J. Sun, X. Hu, X. Huang et al., "Inferring consistent functional interaction patterns from natural stimulus FMRI data," NeuroImage, vol. 61, no. 4, pp. 987-999, 2012.

[13] K. Xie, H. Kuang, and J. Z. Tsien, "Mild Blast Events Alter Anxiety, Memory, and Neural Activity Patterns in the Anterior Cingulate Cortex," PLoS ONE, vol. 8, no. 5, pp. e64907, 2013.

[14] J. S. Liu, "Monte Carlo Strategies in Scientific Computing," Springer, 2001.

[15] C. Meek, "Graphical Models: Selecting Causal and Statistical Models," Carnegie Mellon University, Pittsburgh, PA, 1997.

[16] A. Kumar, and H. D. e. III, "A co-training approach for multi-view spectral clustering," in International Conference on Machine Learning, 2011. 